\title{
Dopa-Responsive Dystonia
}

National Cancer Institute

\section{Source}

National Cancer Institute. Dopa-Responsive Dystonia. NCI Thesaurus. Code C116719.

A genetic disorder in females that presents in early childhood and is responsive to dopamine. It is characterized by clubfeet and Parkinsonian symptoms that may progress from lower to upper extremities witha diurnal pattern, and involuntary muscle contractions and other uncontrolled movements in the lower limbs that worsen with excercise and improve with rest. 